\title{
Pre-processing of On-line Signals in Noisy Environments
}

\author{
Alan McCabe \\ Fern Computer Services Ltd. \\ Belfast, Ireland \\ Email:alan@mymait.com
}

\author{
Jarrod Trevathan \\ School of Maths, Physics and IT \\ James Cook University \\ Email: jarrod.trevathan@jcu.edu.au
}

\begin{abstract}
This paper introduces the Extremum Consistency (EC) algorithm for avoiding local maxima and minima in a specialised domain. The most notable difference between this approach and others in the literature is that it places a greater importance on the width or consistency of an extremum than on its height or depth (amplitude). Short-term, high amplitude extrema can be encountered in many typical situations (such as noisy environments or due to hardware imprecision) and can cause problems with system accuracy. The EC algorithm is far less susceptible to these situations than hill climbing, convolution, thresholding etc., and tends to produce higher quality results. This paper describes the algorithm and presents results from practical experimentation, which illustrates its superiority over other forms of local extrema avoidance in three real-world applications.
\end{abstract}

\section{INTRODUCTION}

This paper presents a new algorithm for avoiding local maxima and minima in specialised environments. The embedding of this algorithm in a number of practical systems has resulted in significant improvements in accuracy when compared with the use of other classical local extrema avoidance algorithms.

Note that throughout this paper the discussion will tend to focus on minima (also known as valleys or troughs) in the interests of brevity. Discussions can trivially be adapted to avoidance of maxima or peaks. Additionally, chiefly for convenience, this algorithm has been given a name: Extremum Consistency or EC.

The underlying problem lies in deciding whether a given extremum represents a "true" extremum. This of course is not a problem new to computer science, but it has never been approached in the particular way described here.

There are several existing algorithms for avoiding local extrema, however none have proven to be sufficiently effective in the specific domain outlined in Section II. The most appropriate of these existing algorithms were implemented in complete systems and the results of these are presented below.

The paper is organised as follows, Section II describes the problem domain and motivation for the algorithm, Section III presents the algorithm and Section IV outlines some of the specific software applications where the algorithm has been successfully applied. Section V presents the concluding remarks and directions for future work.

\section{The Problem Domain - Motivation}

This problem domain is based on iterative improvement strategies that attempt to find minima or maxima while traversing a given stream.

Abstractly, what is required in this domain is for an algorithm to move in the direction of decreasing value until a minimum is reached. Once there, the minimum's location is recorded and, depending on the application, it either proceeds in the opposite direction looking for a corresponding maximum, or "jumps" out of the minimum and begins the process again. This search continues until all of the minima (and maxima if required) are mapped and then processing of these points is performed (see Section IV for more information on the specific applications). Because of this pattern of searching, any false (in a sense, local) minimum encountered will adversely affect the performance of the system, not only because the recorded position is incorrect, but starting the search for the subsequent extremum too early may propagate that error.

The main problem therefore lies in detecting the true (or global) extrema in a volatile and often noisy environment. The EC algorithm is a specific approach to deciding whether a particular extremum represents a true extremum. Figure 1 pictorially shows some of the situations typically encountered

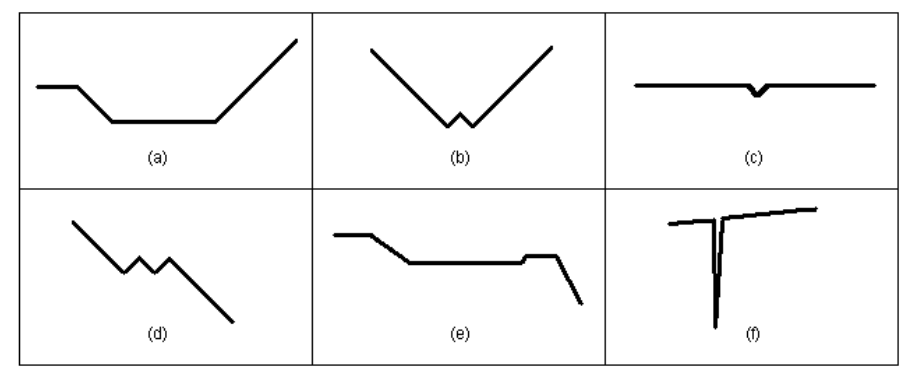

Fig. 1. This figure represents some local minima situations that are typically encountered in processing the input stream. The horizontal-axis represents time and the vertical-axis can represent various stream types such as velocity, direction or temperature. Specifically, (a) contains a valid minimum, (b) contains only a single valid minimum (the appearance of two minima is caused by some noise in the stream, so the second should be ignored) and no others contain any "true" minima, according to our definition (the minima in (c) and (f) are probably a result of noise in this environment, rather than genuine minima, and the same could be said for (d) and (e)). An effective algorithm should reflect this. 


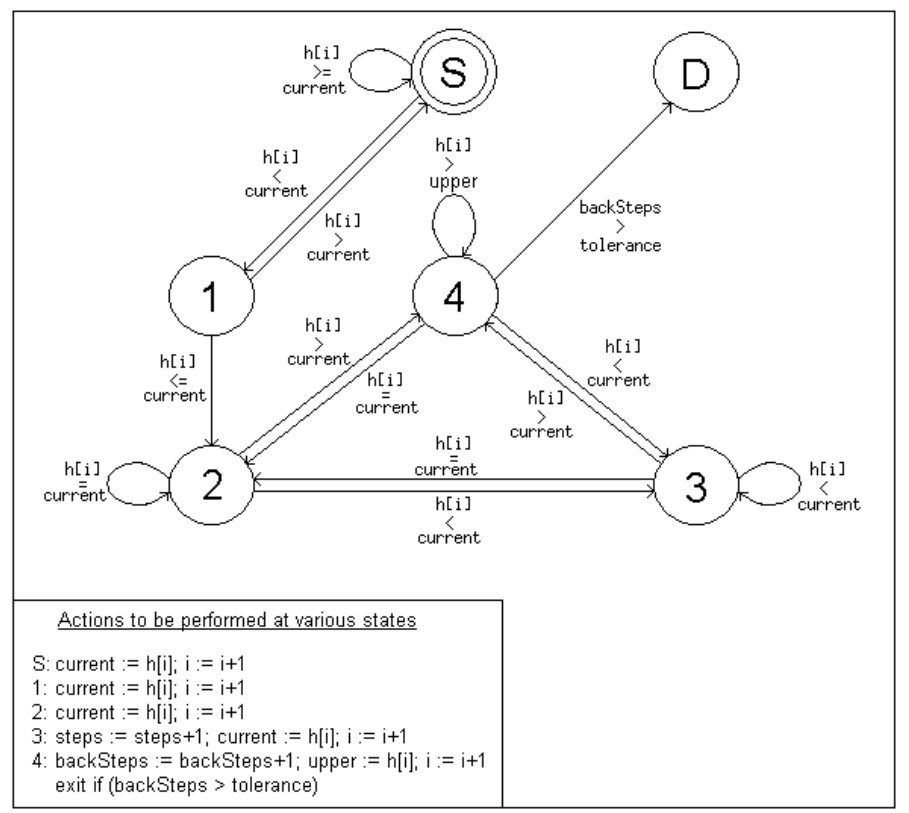

Fig. 2. A finite state machine expressing the EC algorithm for finding the "width" of, or number of "steps" in, the initial downslope of a valley. The movements between vertices (states) are defined by the comparisons between points in the input stream, and are included on the edges in the diagram. Additionally there are actions to be performed when some vertices are reached - these are also included in the diagram. Note that in this table, $h[i]$ refers to the $i^{\text {th }}$ element in the list of stream values $h$. Once the backSteps parameter exceeds the tolerance, the algorithm enters the dead state $D$ and we have the width of the slope in the steps parameter. The width of the upslope is similarly calculable, and the width of peaks can also be found with minimal modifications to the algorithm.

in this environment.

The challenge for this and similar algorithms is to ignore meaningless small fluctuations that appear in the stream, while recording the meaningful fluctuations. The problem lies in distinguishing between the two. Local extrema can enter the data as a result of various aspects, such as quantisation noise, rounding problems or, in handwriting based applications (discussed in Section IV), something as simple as shaky hands or the writing surface itself. These local extrema can be of varying scale, making them more difficult for conventional algorithms to overcome.

\section{The Algorithm}

The algorithm is compact, requiring very little stored data (four integers), no search tree, and is implemented via a series of comparisons done while traversing the surface of the feature space. Additionally, the algorithm is only executed when a potential minimum is encountered so the effect on the efficiency of the overall system is slight.

The major difference between this algorithm and others is that it examines, primarily, the width (or perhaps more accurately the consistency or duration) of the minima. Most other algorithms (such as convolution and thresholding) place more emphasis on the depth of the minima. The use of the term width here differs slightly from an intuitive understanding of the width of a valley. The width of a valley is best explained by considering the initial valley downslope and the following upslope separately. It is defined as the number of "steps" encountered in its traversal where a step, in a downslope, refers to a decrease in height below the value of the current

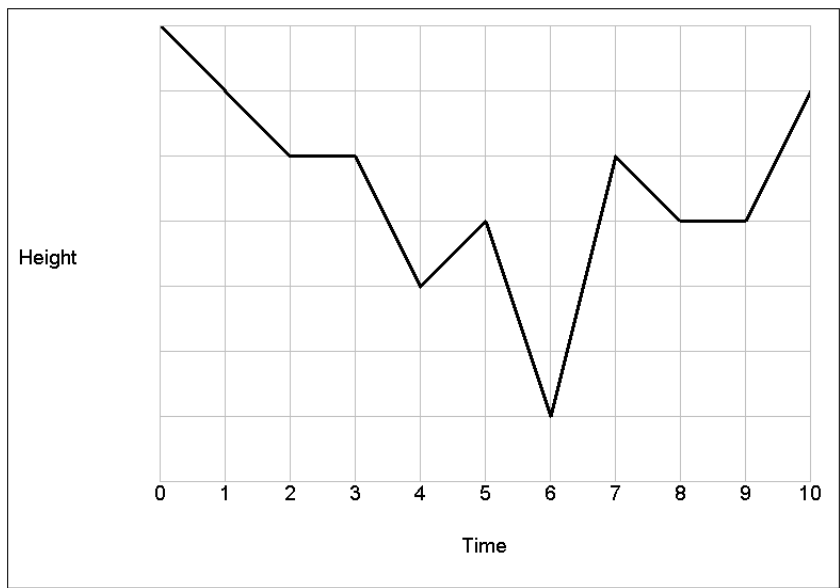

Fig. 3. An illustration of step and width calculation. Valid steps occur between time points 0 and 1,1 and 2,3 and 4 and 5 and 6 , but not 7 and 8 . Backward steps occur between time points 4 and 5, 6 and 7, and between 9 and 10 .

minimum. The more of these decreases there are, the larger the number of steps. This definition can be simply modified to find the number of steps in the upslope. Once these two values are found, the width of the valley is defined as the minimum of the individual width values.

Figure 2 presents a finite state machine illustrating the EC algorithm's operation. The remainder of this section contains descriptions to accompany the illustrations.

Figure 3 illustrates an example of step and width calculation. The term "backward steps" is now also defined as 
movements away from the extremum, beyond the current maximum. For example, in Figure 3, the movement between Time $=4$ and Time $=5$, Time $=6$ and Time $=7$ as well as between Time $=9$ and Time $=10$ can be thought of as taking a backward step. A tolerance parameter determines how many backward steps are accepted before the algorithm terminates. This figure also shows why minimum width is used, rather than mean. Consider the valley that occurs between Time $=0$ and Time $=5$. Using mean width here would erroneously label this as a much larger valley than it really is.

Upon termination we have the value for the slope's width. Tolerance then becomes a significant factor as it represents the amount of time spent looking for a "better" minimum before giving up and accepting the one we have. If the minimum's location is the only information sought (as is often the case, depending on the application), the goal has been achieved and the algorithm ends. If, however, the width of the entire valley is sought (as in the application described in Section IV-A) then the search for the top of the post-valley upslope takes place, starting from the valley floor.

With the width of the valley obtained it is then just a matter of setting a threshold on which width sizes will be considered large enough to constitute a genuine valley (and similarly for peaks). The calculation of the optimal threshold was done during a training phase via brute force experimentation for each application. Results typically worsened considerably for a threshold value of five or over and for all applications in Section IV a threshold value of just two proved to be globally optimal.

The final phase in experimentation involved taking both an extremum's height/depth and width value into account, in an attempt to obtain an even more accurate estimate of true extrema. The simplest and most successful combination method was to simply take the product of the valley width and depth (or height). The result of this was that deeper valleys were now considered better minima than shallow valleys with similar width, which intuitively seems a more desirable effect. In the experiments conducted, this approach consistently provided the best overall results.

\section{SuCCESSFul Applications}

It is worth noting at this point that the algorithm presented in Section III is used essentially as a pre-processing filter, the output of which serves as input to another stage (for example, as part of a signature verification system). The only real way that success of the $\mathrm{EC}$ algorithm is measured is by evaluating the success of the resulting application as a whole. This section briefly discusses some of the application areas in which the EC algorithm has been successfully employed. Further details of these applications can be found in the referenced documents.

\section{A. Direction Based Handwritten Signature Verification}

This was the first project to benefit from the application of the EC algorithm as it relies heavily on extrema detection. It involved the design and development of a dynamic signature verification system [1] which, at its most basic level, tracked the direction of the pen-tip when performing a signature. Peaks and valleys (maxima and minima) were detected in both the horizontal and vertical directions, ordered and converted into a character string (see [1] for more detail).

The initial attempt at valley detection was to implement a naive gradient-descent algorithm that simply traversed in the direction of non-increasing value until the point where a greater value was encountered. This previous (lowest) point was then deemed to be the minimum (note that the surface is only one-dimensional, so there is no choice as to which direction to take when traversing). The problem with this approach was that noise periodically appeared in the stream producing false extrema. This often resulted in the character string becoming somewhat mis-representative of the signature, degrading the whole system's effectiveness.

The overall error rate (sum of false rejection and false acceptance rates) for the first version of the system was $6.9 \%$. This was improved dramatically to $2.9 \%$ with the implementation of the $\mathrm{EC}$ algorithm, with the false rejection rate (the proportion of genuine signatures rejected as forgeries) improved from $4.3 \%$ to $0.9 \%$ and the false acceptance rate (forgeries accepted as genuine signatures) improved from $2.6 \%$ to $2.0 \%$. As mentioned in Section III, the final phase of $\mathrm{EC}$ involved combining the width of an extrema with its "amplitude" (height/depth). The purpose of this being to assign a higher importance to extrema with higher amplitude, versus lower amplitude extrema of similar width. The best combination method was to take the product of the two values, which resulted in a significant improvement of the overall error rate to $2.3 \%$.

In further attempts to improve the error rates, other classical extrema avoidance algorithms were implemented such as thresholding, convolution and simulated annealing. There was also some experimentation with combining convolution with the EC algorithm. This involved using convolution to smooth the input stream before applying $\mathrm{EC}$ to detect the extrema. This approach proved to be more successful than convolution with simple hill-climbing but less successful than EC alone. The reason for this is most probably related to convolution

\begin{tabular}{|l|c|}
\hline Technique & Error Rate \\
\hline Simple Hill Climbing & $6.9 \%$ \\
\hline Thresholding & $17.0 \%$ \\
\hline Simulated Annealing & $24.0 \%$ \\
\hline Convolution and Hill Climbing & $5.3 \%$ \\
\hline EC & $2.9 \%$ \\
\hline Convolution and EC & $3.4 \%$ \\
\hline EC (width $\times$ height) & $2.3 \%$ \\
\hline
\end{tabular}

TABLE I

ERROR RATES USING VARIOUS METHODS OF OVERCOMING FALSE EXTREMA IN A SPECIFIC SIGNATURE VERIFICATION ENVIRONMENT. IF THERE ARE PARAMETERS INVOLVED IN THE OPERATION (SUCH AS CONVOLUTION WINDOW SIZE) THEN THE PARAMETERS PRODUCING THE LOWEST ERROR RATE WERE USED TO GENERATE THE RESULTS. 
smoothing out small but meaningful extrema.

Table I summarises the error rates of the implemented approaches used in the signature verification system. The EC algorithm is clearly superior in this environment.

The other advantage of the $\mathrm{EC}$ algorithm over convolution is execution speed. In a real-time application like signature verification, execution speed can become a serious issue. Empirical experimentation with the signature verification system has found that convolution causes an average slowdown of 20$25 \%$ (depending on system parameters). The number of extra calculations required in the $\mathrm{EC}$ algorithm compared to naive hill-climbing is almost negligible with the slowdown of the signature verification system found to be less than $3 \%$.

\section{B. Velocity Based Handwritten Signature and Password Veri- fication}

The EC algorithm was also used in a handwritten password verification system [2]. The first step in this system (as well as many other handwriting based systems like character recognition algorithms [4]) was to segment the writing stream into its conceptually significant or constituent parts, commonly known as strokes. The strokes are continuous "pen-down" segments of writing bounded by consecutive minima in the pen-tip velocity. The approach then is to extract properties of these strokes and model these properties using a hidden Markov model or neural network.

An approach along these lines was presented previously in [2] and it also made successful use of the EC algorithm. The most naive method of obtaining the velocity minima is a basic gradient-descent algorithm. This was seen as an obvious application for the EC algorithm and it was implemented immediately. A simple gradient-descent implementation was also performed for comparative purposes. Simple gradientdescent produced a total error rate of $2.7 \%$ for password verification compared with $0.79 \%$ using EC with width only, and $0.64 \%$ when using $\mathrm{EC}$ with the product of width and height to do the segmentation. A similar EC implementation was also used as a method of signature segmentation in recent, more extensive studies [3], [6].

\section{Physiology Research - Tracking Fluctuations in Infant Face Temperature}

This physiological research project, initially presented in [5], involved examining fluctuating infant facial temperatures and detecting the exact location of temperature maxima. Without going into excessive detail regarding the project's medical aspects, it is theorised that a climax of increasing facial temperature closely correlates with other physiological episodes. Our task was to accurately determine the occurence of these maxima in real-time.

Initially developed software implemented a simple hill climbing approach to determine the location (in time) of the temperature maxima. These times were correlated with the nearest occurrence of a particular physiological episode and the correlation value, or $p$-value, was 0.072 . That is, the relationship was not significant.
The EC algorithm (width $\times$ height) was also implemented to detect the temperature maxima. The same correlation calculation method was used and the $p$-value improved to 0.001 (highly statistically significant). These results appear to indicate that the $\mathrm{EC}$ algorithm is providing a more accurate estimate of the true temperature maxima.

\section{CONCLusions}

This paper presents a novel local minima and maxima avoidance algorithm. The EC algorithm examines an extrema's width or consistency more so than the actual height. Shortterm peaks/valleys can be encountered in many situations (e.g., noisy environments/hardware inaccuracies) and can cause system accuracy problems. The EC algorithm is far less susceptible to these anomalies than existing techniques and tends to produce higher quality results.

It was shown that the EC algorithm is effective in various practical iterative improvement environments. This paper discussed several specific large-scale applications, and performed a comparison between the $\mathrm{EC}$ algorithm and hill-climbing, convolution, thresholding and simulated annealing. The EC algorithm resulted in a notable improvement over the other approaches in all of the explored applications.

Future work involves comparing the algorithm's effectiveness to other optimisation techniques, along with adapting the algorithm to multi-dimensional space. Should this adaptation be successful, it would be interesting to examine its utility in traversing error surfaces for more effective neural network training. We are also applying the EC algorithm to software bidding agents to enable correct measurements of maximum and minimum prices.

\section{REFERENCES}

[1] McCabe, A. Implementation and Analysis of a Handwritten Signature Verification Technique. Honours Thesis, James Cook University, 1997.

[2] McCabe, A. Markov Modelling of Simple Directional Features for Effective and Efficient Handwriting Verification. R. Mizoguchi and J. Slaney (Eds.): PRICAI 2000, LNAI 1886, pp 801(1-12), 2000.

[3] McCabe, A. Handwritten Signature Verification Using Complementary Statistical Models. Ph.D. Thesis, James Cook University, 2004.

[4] Plamondon, R. and Srihari, S. On-line and Off-line Handwriting Recognition: A Comprehensive Survey. TPAMI, Vol. 22, No. 1, pp 63-84, 2000.

[5] Russell, M.J. and Vink, R. Increased Facial Temperature as an Early Warning in Sudden Infant Death Syndrome. Medical Hypotheses, 2001.

[6] Trevathan, J. and McCabe, A. Remote Handwritten Signature Authentication. In Proceedings of the 2nd International Conference on EBusiness and Telecommunication Networks (ICETE '05), pp 335-339, 2005. 